山्山FANÇAISE

$>\mathrm{DE}$

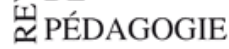

\section{Revue française de pédagogie}

Recherches en éducation

$186 \mid 2014$

Les trajectoires des inspections scolaires en Europe : analyses comparatives

\title{
Les enjeux du rapport aux sciences humaines et sociales dans la formation des ingénieurs en France
}

What's at stake for the humanities and social sciences in French engineering studies

\section{Brigitte Albero et Catherine Roby}

\section{(2) OpenEdition}

Journals

Édition électronique

URL : http://journals.openedition.org/rfp/4405

DOI : $10.4000 / \mathrm{rfp} .4405$

ISSN : 2105-2913

Éditeur

ENS Éditions

\section{Édition imprimée}

Date de publication : 1 janvier 2014

Pagination : 59-73

ISBN : 978-2-84788-639-9

ISSN : 0556-7807

Référence électronique

Brigitte Albero et Catherine Roby, «Les enjeux du rapport aux sciences humaines et sociales dans la formation des ingénieurs en France », Revue française de pédagogie [En ligne], 186 | 2014, mis en ligne le 01 janvier 2017, consulté le 21 décembre 2020. URL : http://journals.openedition.org/rfp/4405 ; DOI : https://doi.org/10.4000/rfp.4405 


\section{VARIA}

\section{Les enjeux du rapport aux sciences humaines et sociales dans la formation des ingénieurs en France}

\section{Brigitte Albero et Catherine Roby}

Les sciences humaines et sociales (SHS), parmi lesquelles l'anthropologie, l'histoire et la sociologie des sciences, ont conduit à relier rationalité des sciences et des techniques et perspectives épistémiques de nature culturelle, politique, économique, éthique. Les dispositifs de formation des ingénieurs intègrent-ils ces connaissances? La compréhension des intrications bio-socio-techniques ne constitue-t-elle pas aujourd'hui l'enjeu principal de ces formations? Dans quelle mesure ces connaissances, théoriquement et empiriquement étayées mais aussi réflexives, permettent-elles la prise en compte des réalités de contexte? Quelle est la place accordée au développement de telles compétences dans les formations des futurs professionnels? Cet article propose de documenter et de discuter l'interrelation entre l'humain et la technique comme enjeu central de la contribution que les SHS peuvent apporter aux formations de hauts niveaux de qualification.

Mots-clés (TESE) : ingénierie, enseignement supérieur, sciences sociales, formation et enseignement professionnels.

L'analyse des dispositifs et des pratiques de formation des ingénieurs en France est peu présente dans les travaux de recherche des sciences humaines et sociales (SHS), notamment en sciences de l'éducation. Pourtant, l'actualité de la formation et des conditions de la pratique professionnelle laisse penser qu'il est pertinent de s'y intéresser, et ce pour plusieurs raisons.

De longue date, les SHS développent des connaissances qui documentent le rapport que l'humain entretient à la science et à la technique, or, lorsque l'on s'attache à étudier empiriquement la place et la fonction accordées aux SHS dans les formations d'ingénieurs en France $^{1}$, force est de constater que l'articulation ne se fait pas. Les affichages de SHS dans les cursus sont majoritairement ceux des enseignements directement liés au monde de l'entreprise et visant des applications utilitaires.

Par ailleurs, depuis la décennie 1970, I'histoire et la sociologie des sciences mais aussi, plus récemment, les disciplines qui analysent l'activité humaine (éducation et formation, gestion et management, information et communication, santé, travail, sports) produisent des connaissances qui tendent à relier rationalité des sciences et des techniques et perspectives épistémiques d'une autre nature (culturelle, politique, économique, éthique). Là encore, l'enquête empirique montre que la formation reste 
souvent centrée sur les acquisitions strictement scientifiques et techniques ou que les enseignements dits d'ouverture au sens large (SHS, culture générale, humanités) ne laissent qu'une place marginale, voire inexistante, à la compréhension des liens entre savoirs savants, savoirs enseignés dans les curricula et projets de société.

Enfin, si l'on s'accorde à penser que l'enjeu principal des formations scientifiques et techniques ne se limite pas à l'acquisition stricte de savoirs et de savoir-faire réduits à ces champs, mais relève également du développement de dispositions à la responsabilité, à l'action stratégique et à la décision, dans la prise en compte réflexive des réalités de contexte et des caractéristiques de l'activité humaine, il apparaît alors pertinent de chercher à savoir quelle est la place accordée au développement de telles compétences dans les cursus, en l'occurrence par l'articulation entre sciences de l'ingénieur et SHS.

En prenant appui sur ces trois raisons qui justifient l'intérêt en sciences de l'éducation pour la formation des ingénieurs, cette contribution ${ }^{2}$ vise à mettre en valeur les enjeux identifiés dans les relations qui s'établissent ou non entre les travaux théoriques produits en SHS sur la problématique du rapport humain à la technique et les contenus des curricula. Ces derniers sont analysés dans le cadre d'une enquête empirique nationale conduite au cours d'une thèse de doctorat (Roby, thèse en cours, voir note 1). Pour ce faire, la première partie met en évidence les apports significatifs et originaux de travaux de SHS qui questionnent les pratiques ordinaires de ces formations. Elle permet de constater qu'il en existe peu sur les interrelations entre SHS et formations d'ingénieurs. La deuxième partie présente de manière synthétique les conditions de construction et les résultats d'une typologie sur la place limitée et problématique des SHS dans les écoles d'ingénieurs en France. En prenant appui sur ces connaissances théoriques et empiriques, une troisième partie propose des pistes de réflexion et d'investigation pour mieux appréhender du côté de la recherche, et mieux exploiter du côté de l'offre curriculaire, les apports des SHS à la formation des ingénieurs.

\section{PRINCIPALES CONTRIBUTIONS SUR LES SHS ET LA FORMATION DES INGÉNIEURS}

Depuis le début des années 1980, des travaux d'historiens et de sociologues, relayés plus récemment par des études en sciences de l'éducation, se sont développés en France sous l'impulsion d'universitaires d'outre-
Atlantique (Fox \& Weisz, 1981 ; Paul, 1985) à propos des institutions scientifiques et techniques et des groupes professionnels tels que les ingénieurs. La plupart de ces travaux n'abordent la formation proprement dite que de manière connexe. Quelques éléments synthétiques d'une revue de la littérature portant sur les travaux des trois dernières décennies mettent en évidence les apports de ces études dans la compréhension de ce milieu spécifique de formation.

\section{Apports des approches socio-historiques sur les institutions scientifiques et techniques}

Dans la décennie 1980, les principales recherches portant directement ou indirectement sur les formations d'ingénieurs en France relèvent de l'histoire ou de la sociohistoire. Elles analysent les pratiques de quelques grandes écoles parisiennes : École supérieure de physique et de chimie (Shinn, 1981), Centrale à Paris (Weiss, 1982), Arts et Métiers (Day, 1991), Ponts et Chaussées (Picon, 1992), Polytechnique (Shinn, 1980; Belhoste, 2003). D'autres travaux ont étudié la naissance des instituts de sciences appliquées dans les universités aux XIX $X^{e}$ et XXe siècles (Grelon, 1989), la naissance de formations particulières telles que celles des ingénieurs en électrotechnique (Badel, 1997) ou des ingénieurs électriciens (Grelon, 1991a, 1991b, 1994) ou encore d'écoles particulières, les Mines à Saint-Étienne (Garçon, 2004), Chimie à Rouen (Bidois, 2007), Centrale à Nantes (Champeau, 2001), industries agricoles et alimentaires à Massy (Vigreux, 2001), I'Université technologique de Belfort-Montbéliard (UTBM) (Lamard \& Lequin, 2006), l'École nationale supérieure d'électricité et de mécanique de Nancy (Birck, 2006; Remoussenard, 2006).

Si de tels travaux ont permis d'élaborer une connaissance sur les origines et le développement des premières formations d'ingénieurs (Picon \& Chatzis, 1992) et de quelques établissements, ils ont permis également d'aborder des thèmes aussi pertinents pour le domaine que la naissance de l'ingénieur moderne (Picon, 1992) et celle d'une technocratie (Belhoste, 2003), la hiérarchisation des formations et leurs relations avec les industries (Grelon, 1989; Garçon, 2004 ; Bidois, 2007 ; Vigreux, 2001; Rollet, 2007), la force de l'ancrage local (Grelon \& Birck, 1998; Birck \& Grelon, 2006; Choffel-Mailfert \& Rollet, 2007) et universitaire (Laurens, 1999). Un article aborde également la question des choix politiques qui orientent les réformes curriculaires dans deux écoles de chimie, organisées autour de configurations symboliques et sociales spécifiques (Bauer \& Cohen, 1981). De telles analyses documentent le rapport que la société entre- 
tient avec la science et la technique depuis le XIX siècle et participent à la compréhension de la genèse de leurs relations dans le monde contemporain.

Malgré l'intérêt de ces apports, la rareté des études et leur éparpillement sont à souligner (Rollet, 2009), invitant à réfléchir à l'importance de susciter des travaux sur la grande diversité de ces écoles qui forment les ingénieurs sous de multiples tutelles (ministères techniques, enseignement supérieur et recherche, avec des écoles internes aux universités ou indépendantes, écoles consulaires, privées, confessionnelles).

\section{Apports des sciences de l'éducation et de la sociologie sur les formations et leurs publics}

Depuis le début des années 1990, les sciences de l'éducation et la sociologie ont conduit des études centrées sur les caractéristiques des formations des ingénieurs. Liés à des situations locales, à des opportunités contractuelles ou à des parcours professionnels particuliers des chercheurs exerçant parfois dans ces écoles, les travaux sont divers dans leurs intérêts, leurs approches théoriques et méthodologiques, ainsi que dans leurs résultats. Ils présentent cependant l'intérêt de mettre en valeur la diversité des thèmes d'enquête permettant d'approcher la variété des réalités liées à ce champ de pratiques : a) en sciences de l'éducation, rôle de la Commission des titres d'ingénieurs (CTI) dans l'organisation des formations (Mirochnikoff, 1990), nature des apprentissages réalisés en formation initiale et dans les parcours professionnels (Robin, 1990) et représentations socioprofessionnelles des élèves ingénieurs (Fraysse, 1996, 2003, 2005), caractère pluridisciplinaire de la formation des ingénieurs forestiers (Cheiko, 2002), formations d'ingénieurs et professionnalisation (Fraysse, 2007; Sonntag, 2007), crise de la formation scientifique des ingénieurs (Bot, 2007), phénomène de mimesis dans la formation des ingénieurs (Lemaître, 2007), questions de formations et place des sciences de l'éducation dans les écoles d'ingénieurs (Sonntag, Lemaître, Fraysse et al. , 2008; Lemaître, 2011); b) en sociologie, processus d'internationalisation et formation des cadres (Lazuech, 1998), relations formation/ emploi (Gaffari, 2003; Bonnet \& Bonnet, 1997), formation initiale à Saint-Cyr (Augé, 2008).

Dans cette variété, certains ensembles thématiques émergent selon les périodes. Ainsi, la création des nouvelles formations d'ingénieurs (NFI) en 1990, dites «filières Decomps ", a-t-elle donné lieu à diverses enquêtes en sciences de l'éducation et en sociologie : émergence d'un autre modèle de la formation et d'une nouvelle profes- sionnalité (Grandgérard, 1996), logiques de cette modernisation (Bousquet \& Grandgérard, 1996), conséquences des évolutions du système productif sur la formation et le travail (Rémy, 1997), rôle des situations professionnelles dans la formation par alternance (Veillard, 2000), assistance et documentation pédagogique en ligne pour des ingénieurs en formation par alternance (Zaid, 2004), trajets professionnels et identitaires entre les fonctions de technicien et d'ingénieur (Rey, 1996; Chaix, 2002, 2007), caractéristiques des nouvelles formations (Roquet, 1999) qui avaient fait, en mars 1996, l'objet d'un numéro spécial de la revue Formation emploi (no 53).

Les problématiques du genre ont également suscité quelques travaux : a) en sciences de l'éducation sur la problématique de la mixité (Stevanovic, 2003) et les représentations des métiers de l'ingénierie (Caillaud, thèse en cours); b) en sociologie, sur l'hypothèse d'une idéologie défensive dans les formes de bizutage (Corbières, 2000); c) en psychologie, sur les stéréotypes du genre et leurs conséquences sur les performances (Smedding, 2009). Ce thème est devenu aujourd'hui l'objet du programme d'études et de recherches Prometea ${ }^{3}$ soutenu par la Commission européenne et coordonné par la Conférence des directeurs des écoles françaises d'ingénieurs (CDEFI), impliquant par exemple l'ENS de Cachan et I'INSA de Lyon en vue de produire une meilleure connaissance des processus de déroulement des carrières.

Plus récemment, de nouvelles thématiques apparaissent et d'autres disciplines contribuent à l'élaboration de connaissances dans le domaine. Ainsi, le développement durable suscite des questions de recherche quant aux relations entre représentations du concept, méthodes pédagogiques en sciences et génie de l'environnement (Lourdel, 2005) ou conduit à interroger différemment les pratiques de formation, par exemple en s'intéressant aux relations entre jeux pédagogiques d'entreprise et intégration des principes d'action du développement durable (Striff, thèse en cours). Le rôle du stage en entreprise dans la formation est étudié en psychologie sociale (Dubruc, 2009).

II faut également signaler dans cette production, le colloque Questions de pédagogie dans l'enseignement supérieur4 (QPES), initié en 2001 par deux écoles d'ingénieurs de Brest, qui donne lieu depuis lors à une manifestation tous les deux ans. Il offre aux professionnels un espace de rencontres et d'échanges pour l'analyse de leurs pratiques et de leurs expériences, en particulier sur des dispositifs de formation qualifiés d'innovants. 
Depuis plus de trente ans donc, diverses disciplines de SHS élaborent des connaissances à propos de la formation des ingénieurs, des écoles et de leurs relations avec l'environnement socio-économique, dont certaines sont susceptibles d'être utiles dans les cursus mêmes de formation des ingénieurs (apports socio-historiques relatifs aux sciences et techniques, questions sociologiques, psychosociologiques, économiques ou de gestion relatives aux organisations et au travail, etc.). La question qui se pose alors est de savoir quelle est la place que les cursus accordent à ces connaissances issues des recherches en SHS, sachant que peu de travaux étudient directement cette question malgré les enjeux qu'elle représente.

\section{Les recherches sur la place des SHS en écoles d'ingénieurs}

La question des relations entre connaissances produites en SHS et formation des ingénieurs suscite des interrogations qui se sont exprimées dans de rares colloques et congrès 5 , deux revues, I'une pluridisciplinaire ${ }^{6}$ (1996), l'autre en sciences de l'éducation 7 (1998), un rapport scientifique (Sainseaulieu \& Jammet, 2011) qui, comme souvent, reste limité à un lectorat spécialisé.

Par ailleurs, les éléments de cette revue de la littérature permettent d'ores et déjà de vérifier qu'il existe peu de travaux de recherche relatifs aux SHS dans les écoles d'ingénieurs en France : deux thèses pionnières en sciences de l'éducation (Dufour, 1998; Lemaître, 2001) posent les bases d'une problématisation; deux thèses en sciences politiques étudient, l'une la réforme du corps des ponts et chaussées en s'intéressant à la figure de l'ingénieur-manager et en y analysant les débats sur le sujet dans la période contemporaine (Gervais, 2007), l'autre l'influence de Gaston Berger dans les conceptions des relations qu'entretiennent sciences humaines et sciences de l'ingénieur (Escudié, 2013). Une nouvelle thèse en cours, en sociologie, propose une analyse socio-historique de l'évolution des débats sur la formation dite "non technique» des ingénieurs en France et en Belgique (Derouet, thèse en cours).

Depuis le début des années 1990, quelques articles ont été publiés sur le thème des SHS dans les écoles d'ingénieurs par des enseignants-chercheurs d'Écoles des mines (Nantes, Saint-Étienne) et de génie industriel (Grenoble) ou encore d'INSA (Lyon, Strasbourg). Les deux seuls ouvrages connus sur ce sujet sont ceux de Lemaître (2003) et de Faucheux et Forest (2007). Directement ou indirectement, ces travaux s'inscrivent tous dans le débat sur la place et la fonction des SHS dans les écoles d'ingénieurs. Ils montrent que leur avenir reste ouvert pour peu qu'elles soient capables d'argumenter leur légitimité auprès des élèves ingénieurs dans des enseignements prenant appui sur les réalités concrètes des situations professionnelles (Minguet, 2001). Cette légitimité devrait consolider la pertinence et l'efficience des SHS dans ce type de formations, apportant une capacité de médiation entre les pratiques technico-économiques et la réflexion éthique (Vinck, 2007). C'est ainsi qu'une formation qui se donnerait pour visée de préparer des ingénieurs responsables de leurs décisions, au double plan environnemental et social, pourrait voir dans l'interdisciplinarité une ouverture possible aux échanges et à la coopération entre une diversité de sciences (Gondran, 2004). Cette perception de l'intérêt de l'interdisciplinarité entre SHS et sciences de l'ingénieur est aussi celle qui naît du rôleclé de la conception dans des processus qualifiés d'innovation qui ne se limitent pas à la stricte application des sciences (Chouteau, Forest \& Nguyen, 2011). Enfin, dans l'univers aussi diversifié que contrasté de ces écoles, la question des recherches en SHS dans les écoles d'ingénieurs a fait l'objet d'un colloque ${ }^{8}$ à l'INSA de Lyon en 2006, au cours duquel les expériences de huit écoles ont été présentées (Faucheux \& Forest, 2007).

Malgré l'importance des enjeux que représente le développement des connaissances sur la formation des ingénieurs et malgré l'intérêt des connaissances déjà produites, on ne peut que constater la rareté de la production en SHS dans le domaine. De manière symétrique, il a paru intéressant de vérifier la place que les formations d'ingénieurs accordent actuellement en France aux connaissances produites par les SHS, qu'elles soient spécifiques (références citées ci-dessus) ou qu'elles portent sur d'autres thèmes susceptibles d'intéresser la formation des ingénieurs.

\section{FORMATION DES INGÉNIEURS ET SHS : UNE RELATION PROBLÉMATIQUE}

Pour approcher les relations que les écoles d'ingénieurs entretiennent avec les SHS, le choix a été fait, dans une première étape d'enquête (2010-2011), d'étudier les programmes de formation tels que les écoles les publient sur leur site internet, en considérant que le curriculum peut être analysé comme un objet socialement construit dans un processus d'élaboration et de négociation qui mobilise des ressources humaines et matérielles, mettant ainsi en jeu des intérêts divers parfois 
divergents, des conflits de valeurs et des rapports de force (Forquin, 2008).

La méthodologie ayant été précisément explicitée par ailleurs (Roby \& Albero, 2013), le choix a été fait dans cette contribution de n'en communiquer que les éléments principaux indispensables à la compréhension du propos (voir annexe).

Si l'on s'en tient aux résultats de la première partie de l'enquête (Roby, 2011), ils tendent à montrer que les affichages dans les cursus relatifs à des enseignements ou modules de formation proches des SHS sont, dans la plupart des cas, directement liés au monde de l'entreprise et visent des applications utilitaires. II a pu être vérifié a posteriori que ces résultats sont cohérents avec ceux obtenus à l'échelle européenne, dans le cadre du projet HELENA (Higher education leading to Engineering and scientific carrers) (Godfroy, 2013). Les affichages de ces enseignements liés au monde de l'entreprise sont souvent proposés sous l'appellation générique de «connaissance de l'entreprise » ou encore de «sciences de l'entreprise » et recouvrent une grande diversité d'approches. La gestion de projet, le management, parfois la sociologie des organisations, mais aussi les techniques d'expression, de communication, voire d'animation de réunion y occupent une place récurrente.

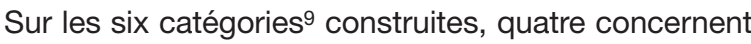
des situations où des savoirs sont potentiellement issus des SHS mais ne s'y réfèrent pas explicitement $(\mathrm{C} 1, \mathrm{C} 2)$ et n'en citent ni les sources ni les ressources (C1). Les modules sont juxtaposés aux enseignements de sciences et techniques de spécialité des ingénieurs sans relation avec eux, ils peuvent être optionnels et sont souvent pris en charge par des professionnels d'entreprises partenaires (C1) et/ou par des enseignants vacataires (C2), dans certains cas, ils sont coordonnés par un permanent $(\mathrm{C} 2, \mathrm{C} 3$, C4). L'accent est mis sur la formation scientifique et technique, les apports relatifs aux SHS relevant d'un «supplément d'âme» visant à sensibiliser les futurs professionnels sur des thèmes différents : le projet personnel et professionnel ainsi que la connaissance de l'entreprise et de ses métiers (C1); une culture générale, un ensemble de qualités professionnelles et des thèmes de droit, d'économie ou de gestion (C2, C3); l'ouverture sur le monde contemporain, les lettres et les arts, parfois les SHS en tant que sciences (C3); les perspectives axiologiques du métier (déontologie, éthique, enjeux) et le développement personnel des étudiants en termes d'exemplarité des conduites professionnelles et personnelles (C4). Dans ces deux catégories (C3 et C4), il s'agit principalement de garantir la polyvalence et l'adaptabilité des ingénieurs, leurs qualités relationnelles, et d'établir leur légitimité sociale, gages de leurs évolutions de carrière. Le profil dominant du professionnel visé est donc tantôt plus technique (C1), plus managérial $(\mathrm{C} 2, \mathrm{C} 3)$ ou plus citoyen (C4) avec une incitation à s'engager dans des activités associatives au sein des écoles ou de projets humanitaires.

Seules deux catégories sur six accordent une place explicite aux enseignements des SHS (C5, C6), mais aussi aux lettres et aux arts (C5) en les reliant à des travaux de recherche au sein même des écoles. Les intervenants sont des enseignants-chercheurs universitaires rattachés à des laboratoires de recherche dans des équipes associées (C5, C6). Les enseignements portent sur l'économie, l'histoire et la philosophie des sciences, la géopolitique, la sociologie, la psychologie, les sciences de gestion et les sciences politiques (C5). Ils peuvent également dépasser les perspectives strictement disciplinaires pour étudier les sciences et les techniques en tant que phénomènes complexes et systèmes d'interrelations entre dimensions économiques, sociales, politiques, scientifiques, culturelles (C6). Cette perspective intégrative des connaissances engage la construction d'approches interdisciplinaires relatives au «cœur de métier » dans les pratiques professionnelles des ingénieurs, orientées par une autonomie de pensée et un esprit critique constructif. Les enseignements disciplinaires (économie, ergonomie, sociologie par exemple) peuvent ainsi être intégrés dans des projets d'ingénieurs placés sous la double responsabilité des enseignantschercheurs de SHS et de sciences et techniques de spécialité des ingénieurs (C6).

Cette catégorisation ne vise bien évidemment pas à classer les écoles mais à clarifier les divers positionnements existants dans la période contemporaine. Les positionnements technologiques $(\mathrm{C} 1, \mathrm{C} 2)$ sont repérés depuis longtemps en opposition aux écoles généralistes; des enseignements potentiellement relatifs aux SHS, à visée pratique et opérationnelle, y ont progressivement été introduits depuis les années 1990, liés à des évolutions de la société ${ }^{10}$. Les autres positionnements possibles sont moins connus et nécessitent d'être nuancés. Par exemple, il est intéressant de constater que certaines catégories regroupent plutôt les écoles privées confessionnelles qui recrutent au niveau bac (C4) ou plutôt les grandes écoles généralistes (C5); elles permettent également de différencier des stratégies de placement dans la hiérarchie implicite qui valorise les écoles généralistes (C3) ou, plus rarement, des stratégies qui articulent une ambition épistémique, axiologique et politique (C6). 
Cette analyse des curricula tels qu'ils sont exposés sur les sites internet met en valeur la place problématique accordée aux SHS. Si, dans quatre catégories sur six, elles n'y apparaissent pas en tant que telles et si la référence à l'entreprise y domine, celle-ci ne semble que peu mise en perspective dans les différentes disciplines dont elle est l'objet et qui relèvent pourtant de la micro-économie, des sciences de gestion, de la sociologie, de la psychosociologie, de l'ergonomie ou encore des sciences du travail. Dans les cursus affichés par les écoles de ces catégories, et à quelques exceptions près, les SHS sont marginales et occupent des fonctions de sensibilisation, d'ouverture, de complément de formation sous forme de culture générale et/ou de formation morale. Avec des volumes d'enseignement de l'ordre de $15 \%$, dans quatre catégories sur six, les SHS sont totalement séparées des enseignements consacrés à l'acquisition des savoirs disciplinaires relatifs aux spécialités techniques. Les SHS ne sont explicitement citées que dans deux catégories sur six : 1) en tant que secteur de connaissance propre à enrichir une formation intellectuelle au même titre que les lettres et les arts (C5); 2) plus rarement, en tant que disciplines intégrées dans le cursus de formation sur des thématiques de formation interdisciplinaire (C6).

Compte tenu des enjeux que représente l'intégration des connaissances produites par les SHS dans la formation de professionnels fortement exposés à la complexification du monde contemporain ${ }^{11}$, la partie suivante tente d'expliciter quelques pistes de réflexion mettant en valeur l'intérêt d'une meilleure intégration des apports des SHS dans la formation des ingénieurs.

\section{SHS ET FORMATION DES INGÉNIEURS : PISTES DE RÉFLEXION ET D'INVESTIGATION}

Au regard des relations problématiques que SHS et formation des ingénieurs entretiennent entre elles, quatre pistes de réflexion transdisciplinaire peuvent être dégagées en tant que secteur d'investigation épistémique et thème de formation. Ces pistes sont susceptibles d'articuler à la fois des intérêts de connaissance et d'action : connaissance produite par le travail d'étude et d'enquête des SHS mais aussi par le travail d'élaboration permanente des métiers de l'ingénierie et du secteur de formation sur les modalités de transmission des savoirs et de l'expérience; l'action de production, de diffusion et de transmission de connaissances à la fois des SHS et des écoles d'ingénieurs selon des styles différents.

\section{Conceptions culturelles des relations entre technique et société}

Sous des appellations diverses (idéel, pré-conceptions, représentations, schèmes, etc.) et des focalisations différentes (anthropologie, psychologie cognitive, sociale, psychosociologie, sociologie clinique, etc.), nombre de travaux en SHS mettent en valeur un principe général : les conceptions de l'action influent sur les modalités de l'action qui à leur tour modifient les conceptions.

Dans le domaine spécifique de l'ingénierie, l'enjeu de la dimension culturelle de la technique recouvre un important thème de travail transdisciplinaire. Des travaux antérieurs $^{12}$ (Albero, 2004, 2010) ont conduit à rappeler que plusieurs disciplines de SHS, en particulier l'anthropologie et la socio-histoire, documentent cette dimension de la technique souvent minorée au profit d'une conception instrumentale. Pourtant, la technique entendue comme fabrication d'artefacts et faire efficace est anthropologiquement constitutive de l'activité humaine dans sa complexité, au même titre que le langage (Leroi-Gourhan, Simondon). L'être humain vit dans une interrelation permanente avec son milieu, médiatisée par la technique (Leroi-Gourhan, Simondon, Mauss, Haudricourt). Elle fait donc partie intégrante du milieu, notamment par les objets produits, et contribue à son organisation, tout comme à son évolution (Leroi-Gourhan, Simondon, Mauss, Haudricourt, Parain, Gille). II a pu être montré (Albero, 2010) qu'en accord avec cette analyse de l'activité technique, la philosophie, en particulier la philosophie allemande d'après-guerre (Heidegger, Habermas, Joas), a mis en évidence l'ambivalence des finalités éthiques, susceptibles de servir la meilleure des causes comme la pire. Si l'action orientée par la seule dynamique du développement technique est susceptible d'amplifier des conduites potentiellement destructrices, une finalité éthique peut orienter le développement technique dans le sens d'un projet social constructif susceptible de produire des évolutions bénéfiques (Ellul, Feenberg, Hottois, Mumford, Sclove).

Dans ce même paradigme, qualifié par ailleurs d'approche culturelle (Albero, 2010), d'autres auteurs, inscrits à l'intersection de différentes disciplines de SHS (anthropologie, histoire, sociologie) ont montré que, dans sa matérialité, la technique est rationalisation du travail humain et des rapports sociaux (organisation, division du travail et coopération rationalisée). Elle est donc également une pratique qui régule les rapports des acteurs sociaux (Mumford, Habermas, Ellul, Roqueplo, Sclove, Salomon). Cette analyse de la technique relie des dimen- 
sions habituellement perçues comme séparées : scientifiques, techniques, économiques, industrielles, sociales, politiques, éthiques. Neutralité et instrumentalité de l'efficacité technique sont alors analysées en tant qu'idéologie, voire en tant qu'erreur épistémique (Leroi-Gourhan, Haudricourt, Castoriadis, Habermas, Ellul, Feenberg, Sfez, Didier). Plus récemment, la socio-histoire des sciences et des techniques (Callon, Latour, Pestre, Prigogyne \& Stengers, Bensaude-Vincent) et celle des processus technocratiques (Belhoste, Letté, Henry) montrent comment certaines professions scientifiques (ingénieurs, experts) sont susceptibles d'exploiter une technique en lieu et place d'une pensée sociale et politique, en se coupant d'une technologie en tant que science humaine (Haudricourt).

Dans une discipline telle que la psychologie, Rabardel (1995) a montré la différence radicale entre approche technocentrée et anthropocentrée dans l'analyse de l'activité humaine instrumentée et dans les développements techniques auxquels elle conduit. Cette différence est symétrique de celle mise en valeur en sociologie (Gras, Bourrier, Boullier, Vinck, Ségrestin) et en information et communication (Flichy, Perriault, Scardigli) entre logique de conception et logique d'usage. Ces orientations de recherche montrent comment la technique peut se mettre au service de l'activité humaine, rendant performantes ses réalisations ou, au contraire, soumettre l'humain à des logiques d'action qui ne sont pas anthropologiquement les siennes (chronobiologie, modes de raisonnement, modalités de la décision, formes d'échanges, langages, valeurs, capacités neurobiologiques).

Ce rapide panorama, nécessairement lacunaire compte tenu de sa visée synthétique, permet cependant de montrer en quoi ces domaines de connaissance devraient pouvoir intéresser un ingénieur contemporain et pourquoi sa formation devrait pouvoir s'y enrichir d'un positionnement réfléchi dans et sur la société, une manière de rechercher des solutions techniques et de contribuer aux prises de décisions à enjeux, une réflexion critique sur la pratique professionnelle experte. L'intégration de tels apports produits par les SHS relève d'une réappropriation par les acteurs du domaine d'une culture de la technique en tant que science humaine, art et métier, intégrée dans un système bio-socio-technique (Akrich, Calame, Linard, Stiegler).

Les écoles d'ingénieurs ont pour vocation de diffuser la culture scientifique qui, en mettant en valeur la pluralité des pensées et des conceptions au sein de débats étayés et rigoureux, tout à la fois scientifiques et sociaux ${ }^{13}$, relève du fonctionnement démocratique auquel aspirent les sociétés (Jollivet, 2011). De la même manière, les écoles d'ingénieurs pourraient aujourd'hui se réapproprier une culture technique reconquise dans le contexte d'une réflexion sur les finalités et les conditions sociales des développements technologiques des sociétés humaines contemporaines. Ce mouvement pourrait s'accompagner d'un changement de perspective dans la formation, en aidant les élèves ingénieurs à passer de l'apprentissage d'une analyse fonctionnelle réalisée à une échelle locale à l'apprentissage d'une analyse compréhensive prenant en compte le contexte de l'instrumentation, les acteurs sociaux concernés et les évolutions possibles dans le temps. Cette démarche pourrait assurer une compréhension de la technique non plus comme simple moyen, mais comme modalité humaine de rapport au monde (Heidegger, Baudrillard, Castoriadis) relevant d'un projet social et politique (Sclove). Concrètement, l'enjeu pour les établissements est d'inscrire un tel projet dans les curricula des enseignements (thématiques transdisciplinaires), dans les modalités mêmes de la formation (autodocumentation, coopération, controverses, cercles d'études) et dans l'accompagnement des apprentissages (études de cas, débats, forums, engagements citoyens) qui nourrissent ce processus de réappropriation, étayent ce type de positionnement, documentent ces techniques d'analyse.

\section{Hiérarchie des disciplines et intérêt des approches inter- et transdisciplinaires}

Dans une perspective de production de connaissance et de formation aux métiers de l'ingénierie, le rapport aux objets techniques gagne donc à s'inscrire dans un mode de pensée étayé et réflexif, tant ceux-ci influent sur les représentations et la conduite des activités, en configurant en grande partie les environnements de travail. De la même manière, la classification des disciplines dans la culture scientifique et technique s'impose comme un allant de soi, alors qu'elle est le fruit d'un construit sociohistorique (Audren, 2006). Quelques éléments fournis dans ce passage de manière synthétique à titre d'exemple tentent de le montrer.

Si les sciences physiques se développent depuis le $\mathrm{XVI}{ }^{\mathrm{e}}$ siècle sur des bases mathématiques, les SHS ne se structurent que depuis la fin du XIXe siècle avec une institutionnalisation tardive au milieu du $x x^{e}$ siècle. Les premières sont devenues le modèle de la science, alors que les secondes n'ont cessé de le questionner. Dans les faits, les relations entre ces domaines de connaissance contribuent à les structurer : celui des mathématiques et 
de la physique en répondant aux besoins de la société et en se développant à partir de questions posées par les SHS (Martin, 2002); celui des SHS en se rapprochant pour partie des méthodes des sciences de la nature (empirisme, expérimentalisme, mathématisation, modélisation). Pourtant, c'est bien la classification sociale des sciences qui réifie le plus efficacement la séparation entre sciences de la nature et SHS, installant une distinction qui annule les rapports qu'entretiennent de fait les sciences avec les mathématiques, la littérature et les arts. Le jeu des réputations des sciences les unes par rapport aux autres en fonction de leur proximité ou de leur éloignement avec les mathématiques contribue également à cette réification. Ainsi, quand, à la fin du XIXe siècle, l'économie abandonne le qualificatif politique, empruntant ensuite les concepts de la mécanique au début du xxe siècle (Le Gall, 2002a), elle augmente son prestige, facilitant ainsi sa diffusion dans les écoles d'ingénieurs, loin de la science sociale des ingénieurs (Le Play, Savoye \& Audren, 2008) et bien loin des SHS. Économie industrielle et économétrie font ainsi partie des enseignements dans les cursus de formation des ingénieurs depuis lors (Le Gall, 2002b). Dans cette même période, les hauts fonctionnaires sont plus proches des théories économiques que les universitaires, et les économistes ingénieurs ont largement contribué à la professionnalisation des économistes experts (Le Merrer, 2011). Malgré l'entrée, à la même époque, de la science sociale dans les deux plus célèbres écoles d'ingénieurs de France, Polytechnique avec Auguste Comte et les Mines avec Frédéric Le Play, les SHS ne se sont guère diffusées dans les établissements au moment de leur expansion tout au long du xxe siècle. Aujourd'hui encore, l'effet d'inertie dans les représentations et les habitus prolonge cette situation, alors même que certaines avancées semblent acquises. Par exemple, le caractère naturalisant de cette classification qui, en instaurant une séparation entre le naturel et le social et par conséquent un dualisme ontologique d'un monde composé d'une réalité naturelle et d'une réalité sociale (Conein, 2001), occulte le fait qu'aucune discipline ne peut dissocier les savoirs qu'elle produit des contextes sociaux, politiques, économiques, éthiques ni des représentations du monde ${ }^{14}$ qui contribuent en partie à leur élaboration (Martin, 2004). La recherche est un acte social situé dans des institutions et dans une histoire. Elle ne se réduit pas à «une activité productrice de réponses à des questions pratiques »; elle est aussi «une entreprise commune de compréhension du monde et des rapports de l'homme à ce monde». Si la production de savoirs est annoncée comme son objectif premier, elle est aussi capacité d'action (Pestre, 2010) et produit en même temps que ces savoirs des transformations technologiques, sociales, politiques, éthiques (Brenner, 2012; Pestre, 2013). Malgré ces avancées, l'ancien dualisme entre rationalité objective et interprétation subjective perdure, et tous les savoirs qui ne relèvent pas directement d'une production inscrite dans les paradigmes des sciences de la nature courent le risque d'être déconsidérés ${ }^{15}$.

L'apparition de nouveaux champs de recherche tels que les sciences cognitives à la fin $d u x x^{e}$ siècle puise autant dans les sciences formelles que dans les SHS ou la philosophie, des pôles de recherches se structurent autour de thématiques interdisciplinaires (nanotechnologies ou épidémiologie). Comment les ingénieurs pourraient-ils rester en dehors de ces préoccupations et de ces nouvelles formes d'organisation du travail scientifique et technologique? Par ailleurs, la fonction critique exercée par les SHS s'avère indispensable dans des contextes de complexification des causes (mondialisation, écologie, gestion des risques, etc.). Elles peuvent donc assurer une fonction de temporisation, de prise de recul, de problématisation face à l'accélération des changements techniques et économiques, centraux dans la formation des ingénieurs.

Documenter cette connaissance du côté de la recherche et placer cette connaissance dans une démarche réflexive du côté de la formation conduisent à remettre en cause des modes de réification de la production même de connaissance, ses modes de diffusion et d'acquisition, voire les modes de réflexion et de prise de décision dans le registre de l'action, en les replaçant au centre du débat démocratique ${ }^{16}$.

\section{Relations entre savoir théorique, ingénierie et action : la part axiologique de la rationalité}

Depuis les années 1970, de nombreuses recherches en anthropologie, philosophie, histoire et sociologie des sciences ${ }^{17}$ montrent que le domaine scientifique et technique relève d'activités sociales intégrées dans un contexte plus large, organisé par des dimensions culturelles, politiques, économiques, éthiques. Bien que fortement structurées en référence à une rationalité objective, distanciée et instrumentée, les démarches scientifiques et technologiques sont, au même titre que les autres activités humaines, orientées par des valeurs (Brenner, Putnam), dépendantes d'un environnement historique et culturel et d'un contexte social et économique.

Le modèle de la science appliquée, qui tend à considérer que les problèmes se résolvent par application de 
théories scientifiques et technologiques, est historiquement structurant pour les formations d'ingénieurs (Schön, 1994). La manière de poser le problème, les conceptions sur lesquelles reposent les conceptualisations mobilisées et le point de vue adopté au moment de l'analyse sont occultés par l'attention accordée au résultat et à l'efficacité immédiate. De plus, en règle générale ces résultats sont attendus en termes de nouveautés techniques, alors qu'il pourrait exister de meilleures solutions, mais dans un autre registre de résolution, politique ou social (Pestre, 2007). Par ailleurs, les processus par lesquels sont construites les décisions prises à propos des moyens à utiliser et des buts à atteindre sont le plus souvent occultés ou peu questionnés, alors que l'on sait aujourd'hui que les méthodes rationnelles des ingénieurs véhiculent des valeurs implicites qui orientent les choix, parfois à l'insu des sujets (Vinck, 2007) et structurent de manière tacite les problèmes et les rôles des différents acteurs (Schön, 1994). À I'heure où les sciences et les techniques fournissent à l'humanité une capacité d'action jamais connue à l'échelle planétaire, l'impossibilité de la maîtrise et celle de l'anticipation des conséquences conduit à penser que la sauvegarde de la diversité dans un horizon démocratique est devenue aujourd'hui vitale pour l'humanité (Pestre, 2007, 2011).

C'est pourquoi la formation des ingénieurs est placée face à la responsabilité qui consiste à développer un modèle de rationalité de l'activité humaine plus approprié aux réalités complexes rencontrées par les professionnels sur les terrains effectifs de leur action : un modèle de science raisonnée intégrant une acception de la science telle que définie par ses aspects théoriques et cognitifs aussi bien que méthodologiques et pratiques, mais aussi éthiques et politiques. Si l'on définit l'ingénierie comme un métier d'interface entre science et technique dans lequel les choix et les arbitrages se font toujours dans un contexte situé, le développement d'une capacité réflexive propre à réguler les multiples relations ${ }^{18}$ qu'il engage apparaît alors indispensable.

\section{Comprendre les ressorts de l'action pour mieux la réguler}

Dans des travaux antérieurs (Albero, 2004), il a été rappelé de manière étayée qu'un lien étymologique relie la technique à l'action accomplie, ce qui donne à la technique une dimension axiologique en attribuant au faire une valeur positive. Le savoir-faire efficace, allié au dynamisme et à l'énergie, fait partie du noyau central des qualités et des valeurs fondatrices des ingénieurs (Grelon, 1995; Giré, Béraud \& Deschamps, 2000) en étant asso- cié à la valeur du progrès matériel (Giré, Béraud \& Deschamps, 2000). Une étude de l'iconographie des plaquettes institutionnelles des INSA a ainsi pu montrer que $75 \%$ des photos sont des images d'action et $50 \%$ de ces photos sont des images d'action technique (Chouteau \& Nguyen, 2007). Cette valorisation du faire efficace par la technique constitue une part de l'identité professionnelle, parfois au détriment d'une valorisation de la réflexion en tant que délibération, considérée comme antagoniste de l'action. Pensée et action sont alors données pour disjointes, alors même que l'analyse de l'activité humaine, quels que soient les courants de recherche (action située, cognition distribuée, cours d'action, approche énactive, pragmatiste, praxéologique, etc.) prouve que pensée et action sont toujours, d'une manière ou d'une autre, reliées.

Dans le secteur de la recherche en SHS qui s'intéresse aux champs de pratiques, notamment professionnelles, le concept de "praticien réflexif » (Schön, 1994) fournit de longue date une piste pour la recherche et pour la formation, en s'accordant aux résultats de recherche sur les caractéristiques de l'activité humaine (Gibson, Mead, Norman, Quéré, Thévenot) et en particulier de l'activité instrumentée (Akrick, Engeström, Linard, Rabardel). Si l'on s'accorde à prendre au sérieux les résultats de ces travaux, la formation à la pratique professionnelle à des niveaux d'expertise (Roqueplo, Salomon, Pestre) conduit à développer des compétences de régulation face à l'incertitude, au changement, à l'inattendu et aux conflits d'intérêts et de valeurs qui limitent sérieusement le champ de la compétence strictement technique. Bien que déjà ancien, le concept de "praticien réflexif» (Schön, 1994) est toujours opératoire dans une période qui n'a pas encore totalement abandonné le modèle, aujourd'hui dépassé, de la science appliquée. II permet de comprendre que le secteur de la formation a la responsabilité de préparer les futurs professionnels à développer des conduites d'analyse des matériaux problématiques d'une situation, complexe par définition, qui mêlent des facteurs d'ordre différent. Dans une telle démarche, les savoirs et les techniques ne sont plus appliqué(e)s mais constituent des ressources réinterprétées en fonction des contingences (contraintes et potentiels) de la situation. De même, l'incertitude n'est plus une menace à réduire mais une donnée féconde, constitutive d'une démarche où la formulation d'hypothèses s'incorpore à l'action dans un dialogue orchestré entre une diversité d'acteurs. Une telle perspective permet de repenser le positionnement de l'ingénieur dans la société en fonction de la manière dont il élabore des solutions, prenant (ou non) en compte les intérêts d'une diversité d'acteurs, et 
contribuant ainsi (ou non) à l'articulation entre savoirs savants et savoirs d'action, concepteurs et usagers, pratiques techniques et action industrielle (Latour, Roqueplo, Vinck), entre projets industriels et projets de société (Callon, Gras, Sclove).

La formation des ingénieurs conduit ainsi à ouvrir les boîtes noires technico-économiques par un travail conjoint entre les disciplines de spécialité des ingénieurs et les SHS (Vinck, 2007) pour produire de nouveaux savoirs ${ }^{19}$, plus adaptés aux situations effectives. Elle conduit également à renouveler les formes pédagogiques $^{20}$, non seulement de la transmission ${ }^{21}$, mais aussi du développement de soi et d'autrui22, voire de la capacité à s'émanciper des règles et des normes ${ }^{23}$. Des formes pédagogiques qui aujourd'hui ne peuvent plus s'arrêter au seuil de la formation initiale des écoles, mais se poursuivent dans des dynamiques de formation continue s'actualisant en permanence au monde contemporain 24 .

\section{ÉLÉMENTS DE CONCLUSION}

Malgré les raisons de diverse nature qui justifient l'intérêt d'un rapprochement, dans la formation des ingénieurs, entre sciences et techniques de spécialité et SHS, le constat d'une relation problématique est doublement étayé par la rareté des travaux des SHS sur les activités professionnelles des ingénieurs et sur leur formation et, de manière symétrique, par la rareté des écoles qui investissent dans la formation interdisciplinaire et le renouvellement des pratiques de formation concernant ces enjeux. Pourtant la prise au sérieux des travaux des SHS qui développent des connaissances pertinentes pour le champ de l'ingénierie, dans une période de complexification, d'accélération et d'internationalisation des problématiques techniques concernant le vivant, qu'il soit naturel et/ou social, pourrait offrir des opportunités et permettre aux écoles de nombreuses initiatives en la matière.
À titre d'exemple, dans la sixième version du document «Références et orientations publié en 2009 par la $\mathrm{CTI}$, les objectifs de la formation sont présentés en trois points : 1) acquisition et maîtrise des connaissances scientifiques et techniques de base (générales, spécialité, ingénieur); 2) adaptation aux champs professionnels nationaux et internationaux (entreprise, innovation et recherche); 3) développement de la dimension humaine et sociétale (personnelle, sociale et environnementale). Ces objectifs renvoient à des qualités reconnues comme pouvant entrer en tension entre elles, par exemple la prise en compte des enjeux de compétitivité et de productivité et la mise en œuvre des principes du développement durable. Ces tensions seraient-elles moindres si la maîtrise des outils et méthodes de l'ingénieur passait par le développement de capacités à poser les problèmes dans toutes leurs dimensions et en tenant compte de leur contexte, avant de chercher à les résoudre techniquement? Ou bien encore si la maîtrise des connaissances scientifiques et techniques comportait une dimension épistémologique? Ce seraient là des capacités que les SHS pourraient aider à acquérir.

Dans leur double dimension, épistémique relevant des savoirs et praxéologique relevant de leur rapport à l'action, ces préoccupations concernent plus largement la problématique de la formation dans le supérieur qui a pour mission de préparer les jeunes générations à des secteurs d'activité d'encadrement et d'expertise, mais aussi les générations en exercice à la transmission de l'expérience et à l'évolution des pratiques.

Brigitte Albero
brigitte.albero@univ-rennes2.fr
Université européenne de Bretagne-Rennes 2,
CREAD (EA 3875)
Catherine Roby
catherine.roby@orange.fr
Université européenne de Bretagne-Rennes 2,
CREAD (EA 3875)

Brigitte Albero etagne-Rennes 2, Catherine Roby CREAD (EA 3875)

\begin{abstract}
ANNEXE
Le corpus réuni par cette étude est constitué des sites internet de la totalité des établissements de formation initiale d'ingénieurs sous statut d'étudiant en tant que mode de formation le plus ancien et le plus répandu ${ }^{25}$ (soit 93,37\% des établissements habilités par la CTI), identifiés à partir de différentes sources officielles ${ }^{26}$. La liste de référence des disciplines constituant les SHS provient des sections $\mathrm{CNU}^{27}$ et a progressivement intégré l'ensemble des appellations utilisées par les écoles pouvant se rapporter de la façon la plus large possible à des SHS en tant que savoirs académiques sur l'humain28. L'analyse progressive des sites, des plus contrastés aux plus similaires, a permis d'éla-
\end{abstract}


borer une base de données contenant l'ensemble des écoles ciblées et l'ensemble des enseignements proposés dans les cursus, ainsi que la présence ou non d'un département regroupant ces enseignements et de recherches en SHS. Une autre base de données a été constituée avec des caractéristiques structurelles des écoles (comme notamment leur localisation, date de création, tutelle, domaine de spécialité, niveau de recrutement). Ensuite, des tris à plats, tris croisés, tests d'indépendance de $\chi^{2}$ avec un ensemble de variables (date de création, tutelle, domaine de spécialité, niveau de recrutement) et des analyses multivariées (ACP) ont été effectués. Cette étape de l'analyse a permis de sélectionner une vingtaine d'écoles sur la base des quatre variables indiquées ci-dessus dont il a pu être vérifié qu'elles influencent tant les affichages des SHS que ceux des départements qui les regroupent ou encore l'existence de recherches en SHS. Sur ces bases, des schémas actanciels ${ }^{29}$ ont ensuite été réalisés grâce une analyse de leurs textes de présentation (école et curriculum de SHS). Cette analyse a débouché sur la construction inductive $^{30}$ de six catégories positionnées sur un continuum entre absence d'apports explicites des SHS et intégration interdisciplinaire avec des sciences de la nature.

\section{NOTES}

1 Étude réalisée dans le cadre d'une thèse en sciences de l'éducation, financée par un contrat doctoral de l'ED SHS 507 : Catherine Roby, Place et fonction des SHS dans les écoles d'ingénieur en France, thèse co-dirigée par Brigitte Albero (Rennes 2) et Denis Lemaître (ENSTA Bretagne, Brest).

2 Cette contribution est le fruit d'une présentation de l'argumentation principale au colloque Les sciences humaines dans les parcours scientifiques et techniques professionnalisants : quelles finalités et quelles modalités pratiques? organisé à Créteil, les 7 et 8 février 2013. Elle tient compte des questions et remarques faites par les organisateurs et le public. Qu'ils soient ici remerciés, de même que les relecteurs anonymes de l'article et la rédaction de la Revue française de pédagogie, pour leurs conseils avisés.

3 En ligne : <http://cordis.europa.eu/project/rcn/74982_en.html> et $<\mathrm{http}: / /$ cordis.europa.eu/result/rcn/46905_en.html> (consultés le 15 avril 2013).

4 En ligne: <http://www.colloque-pedagogie.org/ > (consulté le 15 avril 2013).

5 Colloque Sciences humaines et métiers de l'ingénieur organisé par l'École nationale supérieure des arts et métiers, l'École des Mines de Nancy, l'École nationale des arts industriels de Strasbourg (1994, Cluny). Colloque-congrès Humanités et Grandes Écoles, Conférence des Grandes Écoles, 13-14-15 novembre 1996, École Centrale et INSA Lyon.

6 Revue POUR, «La culture de l'ingénieur », n॰151 (1996). La revue POUR est éditée depuis 1967 par le Groupe de recherche pour l'éducation et la prospective (GREP). Elle propose, sous la forme de numéros spéciaux, un panorama des connaissances et des points de vue sur des questions de société liées à l'évolution économique, sociale et culturelle.

7 Revue Recherche et formation, «Les SHS dans la formation des ingénieurs ", n॰29 (1998), sous la direction d'Odile Bardel-Dedonain et Marie-Laure Chaix. Revue éditée par les éditions de l'ENS de Lyon avec le soutien de l'Institut français de l'éducation (IFÉ). L'IFÉ s'est vu confier une partie des missions nationales de l'Institut national de la recherche pédagogique (INRP).

8 Colloque Les recherches en sciences humaines et sociales dans les écoles d'ingénieurs, organisé les 28 et 29 juin 2006 à l'INSA de Lyon.

9 Par souci de lisibilité, il y sera fait référence dans la suite du texte par la notation suivante : C1, C2, C3, etc.

10 Évolution du marché du travail, concurrence accrue avec les écoles de commerce pour les fonctions managériales, développement des aspects juridiques liés aux activités industrielles, etc.

11 Voir <http://www.cti-commission.fr/lMG/pdf/RetOpfv17postimpres sion.pdf>, p. 29 (consulté le 15 avril 2013).
12 Dans cette partie, sans exhaustivité possible dans le cadre d'un article visant à problématiser le rapport entre SHS et formation des ingénieurs, les auteurs cités en référence le sont à titre d'exemple donnant une orientation sur les travaux du domaine, c'est pourquoi des références exactes de publications ne sont pas fournies, la plupart d'entre elles se trouvant dans les publications antérieures, en particulier : Albero, 2004, 2010.

13 Colloque européen L'inscription sociale de la science (Paris, 5-6 novembre 1998). En ligne : <http://www.unesco.org/most/wscfr. htm> (consulté le 17 avril 2013).

14 En ligne : <http://www.unesco.org/most/wscfr.htm> (consulté le 17 avril 2013).

15 En ligne: <http://www.unesco.org/most/wscfr.htm> (consulté le 17 avril 2013).

16 En ligne : <http://www.unesco.org/most/wscfr.htm> (consulté le 17 avril 2013)

17 Parmi elles : Madeleine Akrich, Barry Barnes, Bernadette BensaudeVincent, David Bloor, Anastasios Brenner, Michel Callon, Karin Cetina-Knorr, Harry Collins, Andrew Feenberg, Michael Gibbons, Alain Gras, Ian Hacking, Monique Linard, Sheila Jasanoff, Bruno Latour, Helga Nowotny, Andrew Pickering, Trevor Pinch, Hillary Putnam, Victor Scardigli, Peter Scott, Simon Schaffer, Steven Shapin, Isabelle Stengers, Langdom Winner, Steeve Woolgar.

18 Entre connaissance savante et prise en compte du contexte; entre modèle conceptuel et situation contingente; entre protocole formel de travail et relations effectives entre acteurs; entre représentations et réalisations effectives; entre solution idéale et résolution préférable; etc.

19 Ergonomie et ergologie, praxéologie des situations professionnelles, socio-histoire des sciences et des techniques, sociologie industrielle, etc.

20 En ré-explorant les potentiels de situations formatives telles que : l'analyse de situations concrètes de travail, l'analyse du fonctionnement des organisations, la recherche sur des thématiques conceptuelles, empiriques ou pratiques; l'entraînement au dialogue, à la controverse, à la gestion de conflits; l'entraînement au travail coopératif et collaboratif; etc.

21 De savoirs, filiations, identités professionnelles, manière de faire, valeurs.

22 Par la capacité à apprendre et à se former à tous les âges de la vie dans une multiplicité de dimensions cognitives, sociales, psychoaffectives, corporelles, existentielles, spirituelles.

23 Pour ouvrir des possibles en termes de : créativité, invention, innovation, résistance à l'autorité. 
24 Ateliers spécialisés, communautés de pratiques, cercles d'études, micro-formations, mutualisations de ressources dans des réseaux d'experts, etc.

25 En ligne : <http://www.cti-commission.fr/IMG/pdf/RetOpfv17post impression.pdf>, p. 12 (consulté le 12 novembre 2011).

26 Journal officiel (JO) de la République française du 10 février 2010 (chaque année, le JO publie la liste des établissements habilités à délivrer un titre d'ingénieur diplômé); sites internet de la CTI, de la conférence des grandes écoles, du centre d'étude des formations d'ingénieurs; guide Hobson des grandes écoles; numéro hors série Le Monde de l'éducation de novembre 2010.

27 En ligne : <http://www.cpcnu.fr/listes-des-sections-cnu> (consulté le 10 janvier 2011).

28 L'appellation SHS est récente dans les écoles d'ingénieurs où les appellations «humanités» et «formation humaine» sont les plus traditionnelles. Tous les termes utilisés dans les cursus pouvant se rapporter à des apports de vulgarisation des SHS, c'est-à-dire à «faire fonction de SHS » ont été scrupuleusement conservés sans modification pour l'étude.

29 En référence à A. J. Greimas (1966a, 1966b) repris par M. Linard $(1989,1994)$ dans la perspective d'une théorisation de l'apprentissage en tant qu'activité humaine instrumentée, l'analyse consiste à identifier les «actants » d'un récit (destinateur-objet-destinataire dans le registre de la "communication », éventuellement les formes de relations entre objet et sujet dans le registre du «désir » ou du "vouloir» et celles entre adjuvant et opposant dans le registre du «pouvoir»). Dans l'analyse conduite, le programme de formation qui s'affiche sur internet est considéré comme un récit. Cela permet de repérer les caractéristiques attribuées : au destinateur (l'école), au destinataire (l'étudiant potentiel), à l'objet (la formation). Ces caractéristiques étant dans ce contexte attachées à des représentations et à des valeurs.

30 Ce travail a permis d'identifier les dominantes de ces discours de présentation et de proposer une catégorisation des écoles. Une relecture complète de tous les textes de présentation des formations de SHS et assimilées de la totalité des écoles a été effectuée jusqu'à saturation des données (Strauss, 1992, 2004). Une hypothèse initiale a permis d'organiser les résultats entre deux pôles contrastés : l'un relevant d'une absence quasi totale d'enseignements des SHS; l'autre relevant d'une intégration en secteurs interdisciplinaires entre sciences des ingénieurs et SHS. L'analyse structurale a permis de construire deux groupements intermédiaires : l'un ne conservant des SHS que leurs apports les plus instrumentaux (techniques de communication ou d'animation de groupe par exemple); l'autre en y sélectionnant des enseignements adaptés au domaine de formation (sociologie du monde rural ou sociologie du travail par exemple). Six grandes catégories se sont ainsi progressivement stabilisées, par approximations successives.

\section{BIBLIOGRAPHIE}

ALBERO B. (2004). "Travaux, interrogations et pistes de réflexion dans un champ de recherche éclaté. Note de synthèse". Savoirs, technologies et formation, n०5, p.11-69.

ALBERO B. (2010). «Penser le rapport entre formation et objets techniques : repères conceptuels et épistémologiques ». In G. Leclercq \& R. Varga, Dispositifs de formation et environnements numériques : enjeux pédagogiques et contraintes informatiques. Paris : Hermès/Lavoisier, p. 37-69.

AUDREN F. (2006). «Explorer les mondes de la science sociale en France". Revue d'histoire des sciences humaines, vol. 15, n०2, p.3-14.

AUGÉ A. (2008). «La formation initiale des futures élites militaires à Saint-Cyr : un dispositif institutionnel en évolution ». Éducation et sociétés, n²1, p. 81-94.

BADEL L. (dir.) (1997). La naissance de l'ingénieur-électricien. Origines et développement des formations nationales électrotechniques. Paris : PUF.

BAUER M. \& COHEN E. (1981). «Politiques d'enseignement et coalitions industrialo-universitaires. L'exemple de deux "grandes écoles" de chimie, 1882-1976". Revue française de sociologie, vol.22, n², p. 183-203.

BELHOSTE B. (2003). La formation d'une technocratie : l'École polytechnique et ses élèves de la Révolution au Second Empire. Paris : Belin.

BIDOIS A. (2007). La formation scientifique et technique dans l'enseignement supérieur à Rouen : 1809-1985. Essai de sociologie historique. Thèse de doctorat, sociologie, université de Rouen.

BONNET R. \& BONNET J. (1997). «Ingénieurs des techniques agricoles : vers de nouvelles relations formation/emploi ». L'Orientation scolaire et professionnelle, vol. 26, n॰3, p. 335-350.
BIRCK F. (2006). «De l'institut électrotechnique de Nancy à l'école nationale supérieure d'électricité et de mécanique de Nancy ». In F. Birck \& A. Grelon (dir.), Un siècle de formation des ingénieurs électriciens. Ancrage local et dynamique européenne, l'exemple de Nancy. Paris : Éd. de la MSH. p.23-88.

BIRCK F. \& GRELON A. (dir.) (2006). Un siècle de formation des ingénieurs électriciens. Ancrage local et dynamique européenne, l'exemple de Nancy. Paris : Éd. de la MSH.

BOT L. (2007). «Éléments d'une crise "post-moderne" dans la formation scientifique des ingénieurs ». Les Sciences de l'éducation - Pour l'Ére nouvelle, vol. 40, n॰3, p.31-57.

BOUSQUET N. \& GRANDGÉRARD C. (1996). «Du modèle des grandes écoles aux formations en partenariat. Quelles logiques de modernisation? 》. Formation emploi, n॰53, p. 75-84.

BRENNER A. (2012). «Les sciences exactes pour quoi faire? Plaidoyer pour une réflexion épistémologique». In M. Conesa, P.-Y Lacour, F. Rousseau \& J.-F Thomas, Fautil brûler les humanités et les sciences humaines et sociales? Paris : Michel Houdiart, p. 185-193.

CALAME P. (1993). Penser l'avenir de la planète, agir dans la complexité. Paris : Fondation Charles Leopold Meyer pour le progrès de l'homme.

CALLON M., LASCOUMES P. \& BARTHES Y. (2001). Agir dans un monde incertain. Essai sur la démocratie technique. Paris : Éd. du Seuil.

CONEIN B. (2001). «Le sociologue dans la nature. Pourquoi pas? ». Revue du MAUSS, vol.17, no1, p. 293-301.

CHAIX M.-L. (2002). «Du technicien à l'ingénieur : les transitions identitaires dans les NFI ». Recherche et formation, n॰41, p. 83-101. 
CHAIX M.-L. (2007). «Alternance et recomposition d'identités d'ingénieurs ». In F. Merhan, C. Ronveaux \& S. Vanhulle (dir.), Alternances en formation. Bruxelles : De Boeck, p. 225-240.

CHAMPEAU V. (2001). De I'I.P.O. à L'E.N.S.M. (1919-1969) : les oscillations d'une école d'ingénieurs en quête de son identité entre industrie et recherche sur les matériaux dans la région nantaise. Thèse de doctorat, histoire, université de Nantes.

CHEIKO M. (2002). Pluridisciplinarité et foresterie : recherche, gestion, pédagogie de projet et formation des ingénieurs forestiers. Thèse de doctorat, sciences de l'éducation, université Lyon 1-Claude Bernard.

CHOFFEL-MAILFERT M.-J \& ROLLET L. (2007). Aux origines d'un pôle scientifique : Faculté des sciences et écoles d'ingénieurs à Nancy du Second Empire aux années 1960. Nancy : Presses universitaires de Nancy.

CHOUTEAU M., FOREST J. \& NGUYEN C. (2011). "Conceptions de l'innovation et formations de l'ingénieur ». Les Cahiers du Musée des confluences, nº 7, p.37-47.

CHOUTEAU M. \& NGUYEN C. (2007). «Les objets techniques dans la "parole" institutionnelle des écoles d'ingénieurs". Communication au colloque Du mode d'existence des objets techniques à l'ère de l'information et de la communication (MEOTIC), organisé à Grenoble les 7 et 8 mars 2007. En ligne : <http://lesenjeux.u-grenoble3.fr/2007-meotic/ ChouteauNguyen/index.html> (consulté le 18 avril 2013).

CORBIÈRES M. (2000). Le bizutage dans les écoles d'ingénieur(e)s : l'asymétrie des sexes comme ressort d'action d'une idéologie défensive. Thèse de doctorat, sociologie, université Toulouse 2-Le Mirail.

DAY C. R. (1991). Les Écoles d'arts et métiers : l'enseignement technique en France, $X I X^{e}-X X^{e}$ siècles. Paris : Belin.

DUBRUC N. (2009). Le stage en entreprise : facteur de développement? Un dispositif de formation structuré par des instruments langagiers : rôle du stage en formation initiale d'ingénieurs. Thèse de doctorat, sciences de l'éducation, université Lumière-Lyon 2.

DUFOUR A. (1998). Les enjeux de l'enseignement de la sociologie dans une École d'ingénieur. Analyse du curriculum de I'Institut supérieur d'agriculture Rhône-Alpes de 1968 à 1994. Thèse de doctorat, sciences de l'éducation, université Lumière-Lyon 2.

ESCUDIÉ M.-P. (2013). Gaston Berger, les sciences humaines et les sciences de l'ingénieur. Un projet de réforme de la société. Thèse de doctorat, sciences de l'éducation, université Lumière-Lyon 2.

FAUCHEUX M. \& FOREST J. (2007). Les recherches en sciences humaines et sociales dans les écoles d'ingénieurs. Paris : Éd. Petra.

FORQUIN J.-C. (2008). Sociologie du curriculum. Rennes : Presses universitaires de Rennes.

FOX R. \& WEISZ G. (1981). The organisation of science and technology in France, 1808-1914. Paris : Éd. de la MSH; Cambridge : Cambridge University Press.

FRAYSSE B. (1996). Évolution des représentations socioprofes- sionnelles des élèves ingénieurs : étude diachronique comparative sur trois départements de I'INSA de Toulouse. Thèse de doctorat, sciences de l'éducation, université Toulouse 2-Le Mirail.

FRAYSSE B. (2003). «Représentations de la formation d'ingénieur au Québec et en France : effet de contexte, évolution(s) possible(s) ». Interlettre Chemin Faisant, n²4.

FRAYSSE B. (2005). «Représentations socioprofessionnelles de l'ingénieur: des outils pour modéliser la complexité». Les Cahiers du C.U.E.E.P.

FRAYSSE B. (2007). "Un dispositif de formation simulé pour professionnaliser les élèves ingénieurs". Les Sciences de l'éducation - Pour l'Ėre nouvelle, n³, p.79-96.

GAFFARI S. (2003). La construction sociale du marché du travail des ingénieurs diplômés. La formation comme dispositif de médiation marchande. Thèse de doctorat, sociologie, université de Nantes et École des Mines de Nantes.

GARÇON A.-F. (2004). Entre l'État et l'usine : l'École des mines de Saint-Étienne au XIXe siècle. Rennes: Presses universitaires de Rennes.

GERVAIS J. (2007). La réforme des cadres de l'action publique ou la fabrique d'un "nouveau " corps des Ponts et Chaussées. Impératifs managériaux, logiques administratives et stratégies corporatistes (fin $d u x x^{e}$ siècle). Thèse de doctorat, sciences politiques, université Lumière-Lyon 2.

GIRÉ A., BÉRAUD A. \& DÉCHAMPS P. (2000). Les ingénieurs : identités en questions. Paris : Harmattan.

GODFROY A. S. (2013). «Décrire et comparer des formations d'ingénieurs interdisciplinaires : enjeux méthodologiques et épistémologiques". Actes du colloque Les sciences humaines dans les parcours scientifiques et techniques professionnalisants : quelles finalités et quelles modalités pratiques? organisé à Créteil les 7 et 8 février 2013.

GONDRAN N. (2004). «De la pluridisciplinaríté pour des ingénieurs généralistes vers une interdisciplinarité à la mesure d'ingénieurs éco-citoyens ". Didaskalia, n²4, p.65-80.

GRANDGÉRARD C. (1996). Émergence d'un contre modèle de formation et nouvelle professionnalité de l'ingénieur. La voie de l'apprentissage. Thèse de doctorat, sciences de l'éducation, université Paris 8-Vincennes-Saint-Denis.

GREIMAS A. (1966a). Sémantique structurale. Recherche et méthode. Paris : Larousse

GREIMAS A. (1966b). «Éléments pour une théorie de l'interprétation du récit mythique». Communications, n॰8, p. 29.

GRELON A. (1989). «Les universités et la formation des ingénieurs en France (1870-1914) ». Formation emploi, n²7-28, p. 65-88.

GRELON A. (1991a). "La Formation des ingénieurs électriciens ». In F. Caron \& F. Cardot, Histoire générale de l'électricité en France (t. 1) : Espoirs et conquêtes, 1881-1918. Paris : Fayard, p. 254-293.

GRELON A. (1991b). «La structuration du réseau de formation des ingénieurs électriciens». In F. Caron \& F. Cardot, Histoire générale de l'électricité en France (t. 1) : Espoirs et conquêtes, 1881-1918. Paris : Fayard, p. 802-848. 
GRELON A. (1994). "La Formation des ingénieurs électriciens ». In M. Lévy-Leboyer \& H. Morsel, Histoire générale de l'électricité en France (t. 2) : 1919-1946. Paris : Fayard, p. 162-216.

GRELON A. (1995). "L'ingénieur catholique et son rôle social». In Y. Cohen \& R. Baudouï, Les chantiers de la paix sociale. Paris : ENS Éditions, p. 167-184.

GRELON A. \& BIRCK F. (1998). Des ingénieurs pour la Lorraine : $X I X^{\ominus}-X X^{\ominus}$ siècles. Metz : Éd. Serpenoise.

HENRY O. (2000). "Henry Le Chatelier et le taylorisme». Actes de la recherche en sciences sociales, vol. 133, no1, p. 79-88.

JOLLIVET M. (2011). "L'avenir de la science est-il dans la société civile?». Natures, sciences, sociétés, vol. 18, n4, p.434-440.

LAMARD P. \& LEQUIN Y.-C. (2006). La technologie entre à l'université : Compiègne, Sevenans, Belfort-Montbéliard. Sevenans : Université de technologie de Belfort-Montbéliard.

LAURENS J. (1999). «Pour une sociologie des institutions scientifiques locales. Le cas de l'Institut de chimie de Montpellier 1889-1957 ». Études héraultaises, n³0-31-32, p. 231-245.

LAZUECH G. (1998). La formation des cadres et ingénieurs : entre savoirs et savoir être, contribution à une sociologie du processus d'internationalisation. Thèse de doctorat, sociologie, université de Nantes.

LEMAÎTRE D. (2001). Formation humaine dans les écoles d'ingénieurs : étude des conceptions contemporaines. Thèse de doctorat, sciences l'éducation, université de Nantes.

LEMAîTRE D. (2003). La formation humaine des ingénieurs. Paris : PUF.

LEMAîTRE D. (2007). «Entre savoirs et identités : le phénomène de mimesis dans la formation des ingénieurs ». Les Sciences de l'éducation - Pour l'Ėre nouvelle, vol. 40, n³, p. 11-29.

LEMAÎTRE D. (2011). «La place des sciences de l'éducation dans les écoles d'ingénieurs : enjeux pour la formation et pour la recherche». In B. Fraysse, Les sciences de l'éducation dans les champs de formation. Quelles mobilisation et légitimation? Paris : L'Harmattan, p. 107-127.

LINARD M. (1989). Des machines et des hommes : apprendre avec les nouvelles technologies. Paris : L'Harmattan.

LINARD M. (1994). «Vers un sujet de la connaissance dans les modélisations de l'apprentissage ». Intellectica, no 2(19), p. 117-165.

LOURDEL N. (2005). Méthodes pédagogiques et représentation de la compréhension du développement durable : application à la formation des élèves ingénieurs. Thèse de doctorat, sciences de l'environnement, université et École des Mines de Saint-Étienne.

LE GALL P. (2002a). «Les représentations du monde et les pensées analogiques des économètres : un siècle de modélisation en perspective». Revue d'histoire des sciences humaines, vol. 6 , n॰1, p. 3964.

LE GALL P. (2002b). «Entre sciences de la nature et sciences humaines : l'économie, science des échanges interdisciplinaires ". Revue d'histoire des sciences humaines, vol. 7, n², p. 311 .
LE MERRER P. L. (2011). "L'affirmation de l'économie comme discipline scientifique : une histoire française particulière ». Tracés. Revue de sciences humaines, hors série n¹1, p. 163-174.

LE PLAY F., SAVOYE A. \& AUDREN F. (2008). Naissance de l'ingénieur social : les ingénieurs des Mines et la science sociale au XIXe siècle. Paris : Mines-ParisTech.

MARTIN O. (2002). «Mathématiques et sciences sociales au $x x^{e}$ siècle". Revue d'histoire des sciences humaines, vol. 6 , n०1, p. 3-13.

MARTIN O. (2004). «France-États-Unis : influences croisées en sciences humaines". Revue d'histoire des sciences humaines, vol. 11, n², p. 3-12.

MINGUET G. (2001). «La formation des ingénieurs aux sciences sociales. Questions de légitimité ». Sociologies pratiques, n०5, p. 1-11.

MIROCHNIKOFF Y. (1990). La formation des ingénieurs en France. Thèse de doctorat, sciences de l'éducation, université Paris 13-Paris Nord.

PAUL H. (1985). From khnowledge to power. The rise of Science Empire in France, 1860-1939. Cambridge : Cambridge University Press.

PESTRE D. (2007). «La gouvernance des sciences en société, la gouvernance des sociétés en science : les tensions entre savoirs, pouvoirs et démocratie ". In Sciences en société au $x x I^{e}$ siècle : autres relations, autres pratiques. Actes du colloque Sciences en société au xxie siècle : autres relations, autres pratiques. Strasbourg : CNRS, p. 41-51.

PESTRE D. (2010). "Dix thèses sur les sciences, la recherche scientifique et le monde social, 1945-2010». Le Mouvement social, vol. 233, n॰4, p. 13-29.

PESTRE D. (2011). «Des sciences, des techniques et de l'ordre démocratique et participatif ». Participations, vol.1, n॰1, p. 210-238.

PESTRE D. (2013). À contre-science : politiques et savoirs des sociétés contemporaines. Paris : Éd. du Seuil.

PICON A. (1992). L'invention de l'ingénieur moderne : l'École des ponts et chaussées, 1747-1851. Paris: Presses de l'École nationale des ponts et chaussées.

PICON A. \& CHATZIS K. (1992). «La formation des ingénieurs français au siècle dernier. Débats, polémiques set conflits ». L'Orientation scolaire et professionnelle, vol.21, $n^{\circ} 3$, p. 227-243.

RABARDEL P. (1995). Les hommes et les technologies. Approche cognitive des instruments contemporains. Paris : Armand Colin.

RÉMY J. (1997). Le travail et la formation des ingénieurs dans un système productif en mutation(s). Le cas des NFI. Thèse de doctorat, sciences de l'éducation, université Toulouse 2-Le Mirail.

REMOUSSENARD P. (2006). «La formation au métier d'ingénieur et ses limites à l'École nationale supérieure d'électricité et de mécanique de Nancy entre 1900 et 1960 ». In F. Birck \& A. Grelon, Un siècle de formation des ingénieurs électriciens. Ancrage local et dynamique européenne, l'exemple de Nancy. Paris : Éd. de la MSH, p. 237-269. 
REY C. (1996). Du technicien à l'ingénieur : trajets professionnels, trajets identitaires. Les stagiaires des Nouvelles Formations d'Ingénieurs (NFI). Thèse de doctorat, sociologie, université François-Rabelais de Tours.

ROBIN J.-Y. (1990). Vers une conception de la formation des cadres. Le cas particulier des ingénieurs. Thèse de doctorat, sciences de l'éducation, université Lumière-Lyon 2.

ROBY C. (2011). «Vers une cartographie des places des sciences humaine et sociales dans les Écoles d'ingénieurs en France ". Doctoriales internationales de l'éducation et de la formation, CREN, Nantes, France, 24-25 novembre.

ROBY C. \& ALBERO B. (2013). «Enquêter sur les formations d'ingénieurs en France : la construction d'une catégorisation sur la place et la fonction des Sciences Humaines et Sociales". Actes du Colloque Les sciences humaines dans les parcours scientifiques et techniques professionnalisants : quelles finalités et quelles modalités pratiques? organisé à Créteil, les 7 et 8 février 2013.

ROLLET L. (2007). «L'école Nationale Supérieure des Industries Chimiques de Nancy et ses partenaires industriels (19201960) ». In M.-J. Choffel-Mailfert \& L. Rollet, Aux origines d'un pôle scientifique. Facultés des sciences et écoles d'ingénieurs à Nancy du Second Empire aux années 1960. Nancy : Presses universitaires de Nancy, p. 205-281.

ROLLET L. (2009). «Peut-on faire I'histoire des pôles scientifiques?». Histoire de l'éducation, no122, p. 93-113.

ROQUET P. (1999). Les nouvelles formations d'ingénieurs : une approche sociologique. Thèse de doctorat, sociologie, université de Versailles-Saint-Quentin-en-Yvelines.

SAINSEAULIEU Y. \& JAMMET T. (2011). Les ingénieurs et leurs compétences non techniques. Lausanne : École Polytechnique fédérale (EPFL).

SAVOYE A. (1988). «La naissance de l'ingénierie sociale». Pour, no119, p. 59-65.

SCHÖN D. A. (1994). Le praticien réflexif : à la recherche du savoir caché dans l'agir professionnel. Montréal : Éd. Logiques.

SHINN T. (1980). Savoir scientifique et pouvoir social. L'École Polytechnique, 1794-1914. Paris : Presses de la fondation nationale des sciences politiques.

SHINN T. (1981). «Des sciences industrielles aux sciences fondamentales. La mutation de l'École supérieure de Physique et de Chimie (1882-1970) ». Revue française de sociologie, n०22, p. 167-182.
SMEDDING A. (2009). Stéréotypes de genre chez les élèves ingénieurs : effets sur les performances cognitives (raisonnement fluide) et étude de leur modulation. Thèse de doctorat, psychologie, université Toulouse 2-Le Mirail.

SONNTAG M. (2007). «Les formations d'ingénieurs : des formations professionnelles et professionnalisantes. Orientations, contenus, contextes". Recherche et formation, n॰55, p. 11-26.

SONNTAG M., LEMAÎTRE D., FRAYSSE B., BECERRIL R. \& OGET D. (2008). "Les questions de formation dans les écoles d'ingénieurs. Un débat reconnu. Une place pour la recherche? ". Recherches \& éducations, n¹, p. 121144.

STEVANOVIC B. (2003). La mixité dans les Écoles d'ingénieurs, le cas paradoxal de l'École polytechnique féminine. Thèse de doctorat, sociologie, université Paris 10-Paris-OuestNanterre-La Défense.

STRAUSS A. (1992). La trame de la négociation : sociologie qualitative et interactionnisme. Textes réunis et présentés par I. Baszanger. Paris : L'Harmattan.

STRAUSS A. (2004). Les fondements de la recherche qualitative : techniques et procédures de développement de la théorie enracinée. Fribourg : Academic Press.

VEILLARD L. (2000). Rôle des situations professionnelles dans la formation par alternance : cas des élèves ingénieurs de production de I'ISTP de Saint-Étienne. Thèse de doctorat, sciences de l'éducation, université Lumière-Lyon 2.

VIGREUX P. (2001). La naissance, le développement et le rôle de l'École nationale supérieure des industries agricoles et alimentaires (ENSIA) 1893-1986. Thèse de doctorat, histoire, université Paris 12-Orsay.

VINCK D. (2007). «La médiation sociologique : entre éthique et pratique, la connaissance». In B. Feltz, P. Goujon, B. Hériard-Dubreuil, S. Lavelle \& W. Lesch, Éthique, technique et démocratie. Louvain-La-Neuve : Bruylant-Academia, p. 253-269.

WEISS J.-H. (1982). The making of technological man: The social origins of French engineering education. Cambridge : MIT press.

ZAID A. (2004). Problèmes didactiques d'une assistance et d'une documentation pédagogique en ligne pour des ingénieurs en formation par alternance. Thèse de doctorat, sciences de l'éducation, ENS Cachan. 